\title{
• 研究报告・
}

\section{猕猴桃倍性混合居群基因组遗传和表观遗传变异}

\author{
颜菱 ${ }^{1,2 \dagger}$, 刘义飞 ${ }^{3 \dagger}$, 黄宏文 $3^{*}$ \\ ${ }^{1}$ 中国科学院武汉植物园, 武汉 $430074 ;^{2}$ 中国科学院研究生院, 北京 100049 \\ ${ }^{3}$ 中国科学院华南植物园, 植物资源保护与可持续利用重点实验室, 广州 510650
}

\begin{abstract}
摘要 植物倍性混合居群的形成和维系常伴随着明显的基因组遗传及表观遗传变异。利用AFLP和MSAP两种分子标记探讨 了中华猕猴桃复合体(Actinidia chinensis)倍性混合居群的遗传变异和结构及其基因组甲基化变异方式。结果表明，该倍性 混合居群具有较高的遗传和表观遗传多样性, 但两者之间没有明显的相关性。种群的遗传多样性与海拔呈显著的负相关 $(P<0.05)$, 但表观遗传多样性与海拔不具显著相关性。AMOVA分析显示, 主要的遗传和表观遗传分化出现在倍性小种内部 (97.65\% vs 99.84\%, $P<0.05)$; 同时, AFLP邻接聚类分析显示二者存在一定程度的倍性相关性, MSAP分析则未显示有明显 的倍性相关性。进一步研究发现, 中华猕猴桃居群的总甲基化程度为 $24.86 \%$, 且多倍体具有更多的甲基化位点变异。该研 究结果为深入探讨狝猴桃倍性混合居群的形成和维系机制奠定了基础。
\end{abstract}

关键词 中华狝猴桃复合体, 混合居群, DNA甲基化, 遗传结构, 多倍化

颜菱, 刘义飞, 黄宏文 (2012). 猕猴桃倍性混合居群基因组遗传和表观遗传变异. 植物学报 47, 454-461.

多倍化是物种形成和进化的主要原动力之一, 在 植物中大约有 $70 \%$ 的被子植物经历了1次或多次多倍 化进程(Masterson, 1994)。多倍体植物通常具有更高 的遗传和形态多样性, 面对变化的生态环境可表现出 更强的生态环境适应能力(Beest et al., 2012)。自然 界的倍性物种或小种通常具有明显的生态地理隔离 分布，但种内或居群内的倍性混合(cytotype mixture) 也较为常见。例如, 对欧洲中部Knautia arvensis agg.自然居群的倍性分析表明, 五倍体通常出现在四 倍体和六倍体混合居群内(Kolár et al., 2009); Marhold等(2010)对东亚地区Cardamine属植物的自然居 群研究也发现了广泛的倍性混合。先前的研究表明, 不同倍性个体的同域分布包含了一些关键的生态遗 传机制，例如定向选择或者平衡选择等。这些机制能 使倍性混合居群呈现动态或者相对稳定的进化状态 (Suda et al., 2007)。此外, 倍性个体内和个体间可呈 现变化的基因流和空间遗传方式(Hardy and Vekemans, 2001; Meirmans et al., 2003)。因此, 对植物 倍性混合居群的研究将有助于了解更多的相关进化 和生态适应性机理。
AFLP (amplified fragment length polymorphi$s m$ ) 是Vos 等(1995)开发的可以在无需预知基因组 DNA序列信息的情况下进行遗传分析的一种分子标 记。该分子标记具有较高的多态效率和较好的通用性, 因而被广泛使用。特别是对于研究多倍体物种, 该分 子标记可以有效地避免读带和数据分析的困难(Aversano et al., 2011)。MSAP (methylation-sensitive amplified polymorphism)是在传统的AFLP分子标记 技术的基础上建立起来的，该分子标记使用对甲基化 敏感的酶探讨基因组甲基化的情况。它除了具备普通 AFLP技术的所有优点外，还可对全基因组范围内胞 嘧啶甲基化程度和状态进行详细的调查分析(ReynaLópez et al., 1997)。例如, Lira-Medeiros等(2010)使 用MSAP方法对盐碱地和河畔2种不同生境的红树林 进行分析发现, 虽然 2 个生境的样本基因组的遗传变 异较小，但却有大量的DNA甲基化变异。Paun等 (2010)通过使用MSAP方法分析发现, 异源四倍体兰 花(Dactylorhiza sp.)DNA甲基化的稳定遗传与部分 环境因子密切相关, 为深入了解多倍体表型进化及适 应性奠定了理论基础。

收稿日期: 2012-02-27; 接受日期: 2012-04-20

基金项目：国家自然科学基金(No.30900119)和中国科学院植物资源保护与可持续利用重点实验室青年基金(No.211006)

$\dagger$ 共同第一作者。

*通讯作者。E-mail: huanghw@mail.scbg.ac.cn 
猕猴桃属 (Actinidia) 隶属猕猴桃科 (Actinidiaceae), 为多年生藤本, 是原产于我国的重要野生果树 资源。世界上大部分野生猕猴桃分布于我国及东亚地 区, 其中我国秦岭以南及横断山脉以东为集中分布区 (黄宏文等, 2000)。猕猴桃属植物的种类较多且形态各 异。该属植物的一个显著特征是种内和种间具丰富的 倍性变异, 包含从二倍体到六倍体(甚至八倍体)的连 续变异(Ferguson and Huang, 2007; 黄宏文, 2009)。 中华狝猴桃复合体(Actinidia chinensis Planch)包含中 华猕猴桃 ( $A$. chinensis var. chinensis)和美味猕猴桃 (A. chinensis var. deliciosa) 2 个变种, 是最具经济价 值且国内外商业化栽培最为广泛的物种(Huang et al., 2004)。先前的研究表明, 该物种复合体具有丰富的倍 性变异 $(2 x-6 x)$, 且在野外呈现连续的地理分布, 倍性 混合居群较为常见(黄韦, 2009; Li et al., 2010)。

本研究以中华猕猴桃复合体(一个较大的倍性混 合居群)为研究对象, 采用AFLP和MSAP两种分子标 记方法比较分析狝猴桃倍性小种种内及种间的遗传 变异方式和结构, 同时研究不同倍性小种的DNA甲 基化程度和变异, 并比较分析与倍性相关的基因组遗 传变异和表观遗传变异的异同, 为深入探讨与猕猴桃 多倍化进程相关的生态遗传适应机制奠定基础。

\section{1 材料与方法}

\section{1 材料采集及其DNA提取}

本研究以湖北宜昌大老岭自然保护区内中华猕猴桃 复合体(Actinidia chinensis Planch)的倍性混合居群 为研究对象, 沿海拔梯度进行样本GPS定位和采集 (图1), 共选取 192株具有不同倍性变异水平(2x: 25 株; 4x: 29株; 6x: 138株)的样本进行分析(未发表资 料)。采集的新鲜叶片使用硅胶干燥保存, 并采用 CTAB 法 (Doyle and Doyle, 1987) 提取基因组总 DNA。经质量检测后 $-20^{\circ} \mathrm{C}$ 保存备用。

\subsection{AFLP及MSAP分析}

AFLP分析及其引物序列参照文献Vos等(1995)所述。 从20对通用引物中篮选出 8 对清晰且多态性稳定的引 物组合进行大规模样本分析(表1)。MSAP分析使用 $E c o R I+H p a l l$ 和 $E c o R I+M s p I$ 内切酶组合对基因组

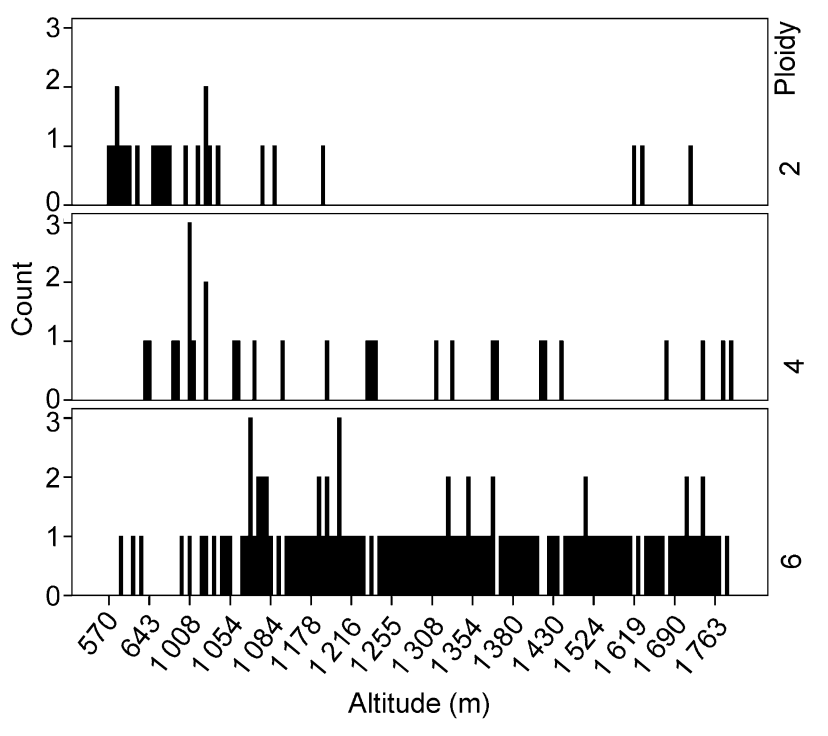

图1 猕猴桃倍性样本的海拔分布

Figure 1 Altitude distribution of different ploidy races of Actinidia chinensis samples

DNA进行酶切。EcoRI接头及其引物序列参照文献 Vos等(1995); Hpall(Mspl)接头及其引物序列参照文 献Xiong等(1999)。随机组合后篮选出条带清晰、多 态性高且稳定的5对引物(表1)。对EcoRI引物5'端进行 $F A M$ 苂光标记, 然后进行选择性扩增并在ABI 3730 $X L$ 自动测序仪上进行条带分型。实验所用接头及其引 物均由生工生物工程(上海)有限公司合成。

\section{3 数据统计与分析}

使用GeneMarker V1.75软件(Hulce et al., 2011)将 AFLP和MSAP的分析结果转化为 0 和1矩阵并进行人 工校对。使用POPGENE V1.32软件 (Yah et al., 2000)计算遗传多样性参数, 包括多态位点百分率 $(P P L) 、 N e i$ 基因多样性指数 $(H)$ 和shannon信息指数 (I)等; 同时利用SPSS16.0软件对遗传多样性指数和 海拔进行相关性分析。利用Arlequin 3.0软件 (Excoffier et al., 2005)进行倍性小种内和小种间的AMOVA分布, 并进行显著性检验。使用NTSYSpc2.1软 件(Rohlf, 2002)进行邻接(neighbor-joining, N-J)聚类 分析, 并对AFLP和MSAP分析进行Mantel检验。最后 使用EXCEL软件统计倍性小种的甲基化水平。 


\section{2 结果与讨论}

\section{1 倍性混合居群的遗传多样性分析}

8对AFLP选择性扩增引物共产生301个位点, 扩增条 带多态性位点百分比为 $99 \%$, 其中六倍体最高, 而二 倍体与四倍体类似(表2)。MSAP分析共使用5对选择性 扩增引物, 通过数据转换共得到222个位点。与AFLP 分析结果类似, MSAP的总体扩增条带多态性位点百 分比也较高, 并且多倍体普遍高于二倍体(表2)。其它 的遗传多样性参数值如表 2 所示。从表 2 可以看出其总 体变化趋势为随着倍性的增加, 遗传多样性指数增 高。进一步对AFLP和MSAP分析产生的遗传多样性参
数矩阵进行相关性分析, 结果显示两种变异之间并无 显著相关性 $(r=0.02, P=0.25)$ 。为了探究遗传和表观遗 传多样性指数与海拔的相关性, 对Nei's遗传多样性指 数和海拔进行了相关性分析, 结果表明遗传多样性与 海拔呈显著负相关 $(r=-0.959, P=0.041)$, 但表观遗传 变异与海拔则相关性不显著 $(r=-0.393, P=0.604)$ 。

\section{2 倍性混合居群的遗传分化及结构}

基于AFLP和MSAP两种数据的AMOVA分析结果表明, 总体的遗传变异和表观遗传变异多存在于倍性小种内 部(97.65\% vs 99.84\%)(表3), 而倍性小种间则较少。

邻接聚类分析结果显示, AFLP与MSAP分析的

表1 AFLP与MSAP选择性扩增引物组合

Table 1 AFLP and MSAP selective primer combinations

\begin{tabular}{lccccccccc}
\hline Labelled & \multicolumn{10}{c}{ Unlabelled primer } \\
\cline { 2 - 9 } & Mse-CAC & Mse-CTA & Mse-CTG & Mse-CTT & Mse-CAT & HM-TCC & HM-TCA & HM-TTC & HM-TGA \\
\hline E-AGC & AFLP\#1 & - & AFLP\#3 & - & - & MSAP\#1 & - & - & MSAP\#5 \\
E-ACC & - & AFLP\#2 & - & - & - & - & MSAP\#2 & - & - \\
E-ACT & - & - & - & - & AFLP\#5 & - & - & - & - \\
E-AGG & AFLP\#8 & AFLP\#6 & - & AFLP\#4 & - & - & - & MSAP\#3 & - \\
E-ACA & - & - & - & - & - & - & - & MSAP\#4 & - \\
E-ATC & - & - & - & AFLP\#7 & - & - & - & - & - \\
\hline
\end{tabular}

表2 中华猕猴桃倍性混合居群AFLP及MSAP分析的遗传多样性水平

Table 2 The genetic diversity level of different ploidy races of Actinidia chinensis based on AFLP and MSAP analyses

\begin{tabular}{ccccccc}
\hline & $\begin{array}{c}\text { Ploidy } \\
\text { levels }\end{array}$ & $\begin{array}{c}\text { Allele number } \\
(\mathrm{Na})\end{array}$ & $\begin{array}{c}\text { Effective alleles } \\
(\mathrm{Ne})\end{array}$ & $\begin{array}{c}\text { Nei's genetic } \\
\text { diversity }(H)\end{array}$ & $\begin{array}{c}\text { Shannon } \\
\text { index }(I)\end{array}$ & $\begin{array}{c}\text { Percentage of } \\
\text { polymorphic loci }(P P L)(\%)\end{array}$ \\
\hline AFLP & $2 \mathrm{x}$ & 1.9203 & 1.5570 & 0.3219 & 0.4797 & 92.03 \\
& $4 \mathrm{x}$ & 1.9203 & 1.5866 & 0.3377 & 0.5003 & 92.03 \\
\multirow{4}{*}{$\mathrm{MSAP}$} & $6 \mathrm{x}$ & 1.9900 & 1.6131 & 0.3556 & 0.5291 & 99.00 \\
& $2 \mathrm{x}$ & 1.9640 & 1.3248 & 0.2268 & 0.3732 & 96.40 \\
& $4 \mathrm{x}$ & 1.9820 & 1.2983 & 0.2117 & 0.3537 & 98.20 \\
\hline
\end{tabular}

表3 中华狝猴桃倍性混合居群AFLP及MSAP的AMOVA分析结果

Table 3 The AMOVA analysis for the cytotype mixture population of Actinidia chinensis based on AFLP and MSAP analyses

\begin{tabular}{lllllcl}
\hline \multicolumn{2}{l}{ Source of variation } & $d f$ & $\begin{array}{c}\text { Sum of } \\
\text { squares }\end{array}$ & $\begin{array}{c}\text { Variance } \\
\text { components }\end{array}$ & $\begin{array}{c}\text { Percentage of } \\
\text { variation (\%) }\end{array}$ & $\begin{array}{c}F- \\
\text { statistics }\end{array}$ \\
\hline \multirow{2}{*}{ AFLP } & Among populations & 2 & 204.963 & $1.235 \mathrm{Va}$ & 2.35 & $0.0235^{*}$ \\
& Within populations & 189 & 9695.177 & $51.297 \mathrm{Vb}$ & 97.65 & - \\
\multirow{3}{*}{ MSAP } & Among populations & 2 & 83.683 & $0.064 \mathrm{Va}$ & 0.16 & $0.0016^{*}$ \\
& Within populations & 189 & 9198.504 & $38.812 \mathrm{Vb}$ & 99.84 & - \\
\hline
\end{tabular}


聚类结果具有明显的区别(图2)。与MSAP分析相比, AFLP分析更明确地展示了与倍性相关的居群遗传结 构。例如二倍体样本多优先聚在一起, 且与四倍体遗 传关系较近(图2A); 相反, MSAP聚类则没有明显的 倍性相关性, 3种倍性个体呈现交互分布的式样(图 2B)。聚类结果与遗传距离矩阵的共表征相关系数分 析表明, AFLP与MSAP分析具有高度一致性 $(r=0.57$ vs $r=0.82, P=0.002)$, 显示聚类结果与遗传距离矩阵 之间有较好的吻合度。

\section{3 倍性混合居群甲基化变异方式}

综合所有样本的 5 对MSAP扩增引物结果可得能辨识
的甲基化位点42 624个, 其中二倍体猕猴桃中检测 到5 550个, 四倍体中检测到6 438个, 六倍体中检测 到30636个。在这些位点中, 检测到的总甲基化率为 24.86\%, 3个倍性小种的甲基化率分别为 $25.24 \%$ (2x)、24.20\%(4x)和 $24.93 \%(6 x)$ 。总体而言, 全甲基 化率高于半甲基化率(15.05\% vs 9.81\%)(表4)。

除了甲基化水平上的不同，不同的倍性小种还 呈现了倍性特异的表观遗传位点。在全甲基化中, 相 对于二倍体, 四倍体表观遗传位点增加了7个, 缺失 了 4 个; 六倍体表观遗传位点增加了11个。在半甲基 化中, 四倍体和六倍体均只有表观遗传位点的增加, 而没有缺失(表5)。

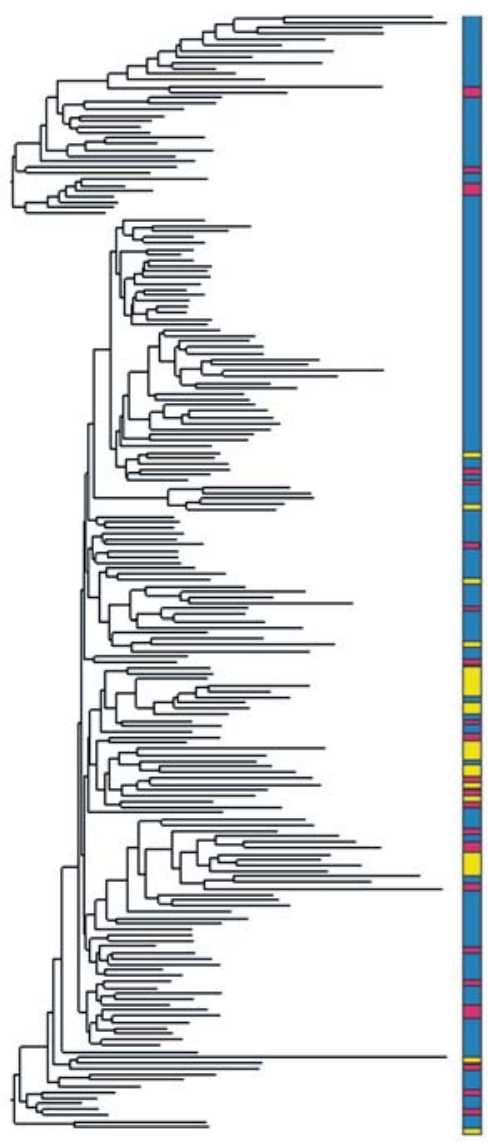

A

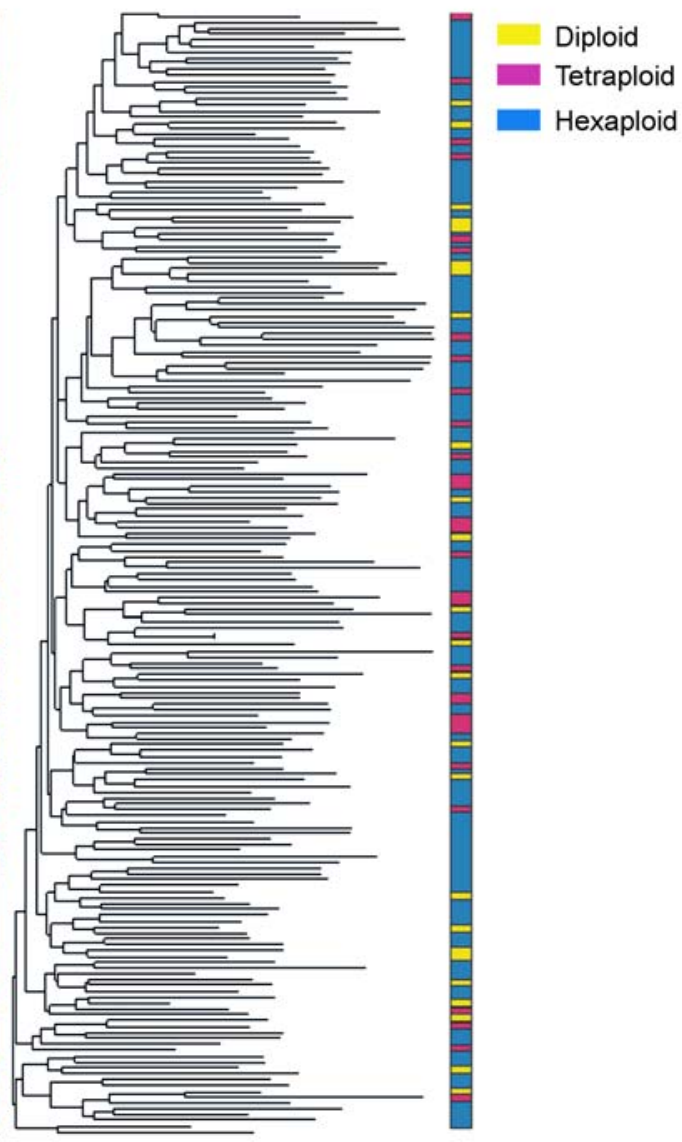

B

图2 中华狝猴桃倍性混合居群基于AFLP及MSAP分析的N-J邻接树聚类图

(A) 基于AFLP分析; (B) 基于MSAP分析

Figure 2 The neighbor-joining (NJ) tree of different ploidy races of Actinidia chinensis based on AFLP and MSAP analyses (A) Based on AFLP analysis; (B) Based on MSAP analysis 
表4 不同倍性水平的中华猕猴桃个体基因组的甲基化水平

Table 4 The DNA methylation levels of different ploidy races of Actinidia chinensis

\begin{tabular}{lcccl}
\hline & Diploid & Tetraploid & Hexaploid & Total \\
\hline Total bands & 5550 & 6438 & 30636 & 42624 \\
Fully methylation bands & 893 & 901 & 4621 & 6415 \\
Hemimethylation bands & 508 & 657 & 3017 & 4182 \\
Total methylation bands & 1401 & 1558 & 7638 & 10597 \\
Fully methylation ratio (\%) & 16.09 & 13.99 & 15.08 & 15.05 \\
Hemimethylation ratio (\%) & 9.15 & 10.21 & 9.85 & 9.81 \\
Total methylation ratio (\%) & 25.24 & 24.20 & 24.93 & 24.86 \\
\hline
\end{tabular}

表5 不同中华猕猴桃倍性小种的甲基化方式和位点差异

Table 5 The difference of DNA methylation patterns and loci of different ploidy races of Actinidia chinensis

\begin{tabular}{|c|c|c|c|c|c|c|}
\hline \multirow[t]{3}{*}{ Ploidy levels } & \multicolumn{2}{|c|}{ Fully methylation } & \multirow[t]{3}{*}{ Unique loci } & \multicolumn{2}{|c|}{ Hemimethylation } & \multirow[t]{3}{*}{ Unique loc } \\
\hline & Diploid & Tetraploid & & Diploid & Tetraploid & \\
\hline & Gain/lose & Gain/lose & & Gain/lose & Gain/lose & \\
\hline Diploid & 0 & - & - & 0 & - & - \\
\hline Tetraploid & $7 / 4$ & - & 2 & $8 / 0$ & - & 5 \\
\hline Hexaploid & $11 / 0$ & $8 / 0$ & 5 & $11 / 0$ & $3 / 0$ & 3 \\
\hline
\end{tabular}

\section{4 讨论}

植物多倍化是一个普遍存在的动态进程。植物多倍化 后, 为了克服基因组加倍带来的冲击, 会迅速产生一 些遗传和表观遗传上的改变, 使其能够更快且更好地 适应新环境 (Soltis et al., 2010; Mayfield et al., 2011)。本研究结果表明, 中华狝猴桃复合体倍性混 合居群不论是在基因组遗传变异还是在表观遗传变 异水平均具有较高的多样性(表2)。先前的研究利用不 同的分子标记(如基因组微卫星和等位酶等)均一致展 现了中华猕猴桃较高的基因组遗传多样性(陈万秋等, 2001; 刘亚令等, 2006; 张田等, 2007), 但尚未见表 观遗传变异的相关报道。本文首次利用MSAP技术, 调查分析了猕猴桃自然居群的表观遗传变异信息。由 于狝猴桃为雌雄异株植物, 广泛的种内及种间基因流 的存在有利于其维持较高水平的遗传多样性(张田等, 2007; Liu et al., 2010)。同时, 多倍化进程相关的基 因组遗传和表观遗传变异也可以促进较高遗传多样 性的出现(Beest et al., 2012)。尽管如此, Mantel检验 分析结果显示, 遗传与表观遗传的变异方式间并无明 显的相关性。这与 $\mathrm{Li}$ 等(2007)对野生大麦(Hordeum vulgare)遗传及其表观遗传变异的研究结果相似, 即 两者无直接联系。此外, 通过对海拔与遗传变异相关
性分析也表明, 种群的遗传多样性随着海拔的升高而 降低, 但对基因组的表观遗传多样性没有显著影响。 这再次证明了AFLP与MSAP分析方式间无显著相 关性。

物种居群的遗传变异和结构是其长期对生态环 境适应性进化的结果(Falk and Holsinger, 1991)。本 研究中, 该倍性混合居群的基因组遗传变异和表观遗 传变异多存在于倍性小种内(AFLP: $97.65 \%$; MSAP: $99.85 \%)$ 。这与先前对该复合体的其它自然居群的遗 传变异分析结果相类似(刘亚令等, 2008)。由于本研 究选取的尺度较小, 不同倍性个体间可以很容易形成 传粉, 广泛的倍性小种内和小种间的基因流可极大地 促进遗传混合和基因渐渗, 从而导致产生观察到的遗 传和表观遗传变异分布方式。最近对狝猴桃属主要物 种的自然同域分布居群的研究也表明, 虽然存在染色 体倍性差异, 但是基因流却非常广泛(张田等, 2007; Li et al., 2010)。

本研究中基因组遗传聚类图基本上遵从了倍性 相同优先聚类的原则(图2)。其中, 二倍体基本上聚为 一类, 同时与六倍体个体相比, 二倍体与四倍体的关 系较近, 表明二倍体和四倍体间可能存在更近的进化 起源关系或更高水平的基因流。但是, MSAP的遗传 聚类表明, 3个倍性小种间的遗传距离较小且不同的 
倍性个体在聚类图上没有明显的倍性相关性。当生态 环境改变时, 植物可通过基因组甲基化等方式产生相 应表达上的变化以适应新的环境。例如, 在对西班牙 东南部地区的多年生植物Viola cazorlensis进行研究 分析时发现, 在应对食草动物的捕食中, 更多的个体 积极通过甲基化反应对捕食做出反抗性的适应(Herrera and Bazaga, 2011)。而Gao等(2010)在对Alternanthera philoxeroides的自然生境及控制生境的转 化实验中以及Paun等(2010)在对兰花的研究中也同 样发现, 环境因子可直接调控表观遗传变异, 从而产 生适应性的变化。本研究中, 表观遗传变异与海拔的 相关性不显著, 这可能是因为除了海拔之外, 复杂生 境中的其它生态因子也在影响DNA甲基化的变异。由 此可以推断, 本研究发现的猕猴桃倍性混合居群并不 明显的MSAP遗传结构可能是其适应相关生态环境 进化的结果。

许多研究结果表明, 基因组多倍化后, 其甲基化 水平均发生了明显改变(Salmon et al., 2005; Keyte et al., 2006)。本研究中, 多倍化后的猕猴桃甲基化水 平也发生了变化, 二倍体总的甲基化水平要高于四倍 体和六倍体, 多倍体的甲基化水平并未随多倍体水平 的升高而升高。其它类似的研究中也观察到这一现 象。例如, 二倍体、三倍体和四倍体西瓜(Citrullus lanatus) 的甲基化水平分别为 $28.00 \% 、 22.90 \%$ 和 $27.37 \%$ (王春国等, 2009); 不同倍性的鼠尾草(Salvia officinalis), 其甲基化水平也各不相同(二倍体为 $43.66 \%$; 三倍体为 $38.87 \%$; 四倍体为 $39.79 \%)(\mathrm{Li}$ et al., 2011)。这些研究均显示没有明显的相关于倍性的 甲基化率的变化。这一现象可能与多倍化进程中的遗 传二倍化现象有关。为了减少基因组加倍后的基因超 量表达, 多倍体基因组的表达水平会被减少到与其二 倍体祖先类似而呈现遗传上的二倍化现象 ( Li et al., 2007)。本研究进一步得到3个倍性猕猴桃小种的总体 甲基化水平为 $24.98 \%$; 与其它物种如多倍体西瓜 $(28.0 \%)$ (王春国等, 2009)和半夏 (Rhizoma pinelliae)(24.3\%)(薛梅等, 2008)相比, 中华猕猴桃复合体 的甲基化水平适中。另外, 本研究还发现, 与多倍体 相比, 猕猴桃二倍体具有更多的特异位点。因此, 在 中华狝猴桃基因组加倍的过程中, DNA甲基化的变异 可能是其适应性进化和倍性同域共存的关键内在机 制之一。
综上所述, 本研究结果表明, 中华猕猴桃倍性混 合居群具有较高的基因组遗传变异和表观遗传变异, 且多样性水平在一定程度上相关于倍性水平。同时发 现, 该居群的遗传和表观遗传分化主要发生在倍性小 种内部，而倍性小种间没有明显的基因交流阻碍。此 外, 邻接聚类分析显示, 中性遗传数据结果与倍性相 同优先聚类的原则完全吻合, 而在表观遗传水平上, DNA甲基化明显影响了倍性个体间的聚类关系。另 外, 本研究还发现, 该倍性混合居群具有适中的甲基 化水平, 且倍性之间差异明显, 这种因倍性不同而变 化的表观遗传变异可能是该倍性混合居群自然选择 和适应性进化的关键机制之一。基于该研究结果及快 速发展的分子生物学技术, 今后应进一步结合相关的 分子标记技术和可能的倍性混合居群的繁殖及生物 学规律深入探讨该倍性混合居群的稳定维系机制, 为 相应猕猴桃多倍体资源的发掘利用提供指导。

致谢中国科学院华南植物园岁立云同志和中国科 学院武汉植物园周锦同志在DNA提取方面给予诸多 帮助, 特此致谢!

\section{参考文献}

陈万秋, 叶新太, 李思光, 薛喜文 (2001). 猕猴桃酯酶同工酶 和过氧化物酶同工酶分析及遗传多样性研究. 南昌大学学 报(理科版) 25, 269-272.

黄宏文 (2009). 猕猴桃驯化改良百年启示及天然居群遗传渐 渗的基因发掘. 植物学报 44, 127-142.

黄宏文, 烡俊杰, 王圣梅, 何子灿, 张忠慧, 李建强 (2000). 猕猴桃属(Actinidia)植物的遗传多样性. 生物多样性 8, 112.

黄韦 (2009). 中华猕猴桃/美味猕猴桃复合体自然居群倍性变 异格局的研究. 硕士论文. 武汉: 华中农业大学. pp. 27-28. 刘亚令, 李作洲, 姜正旺, 刘义飞, 黄宏文 (2008). 中华猕猴 桃和美味狝猴桃自然居群遗传结构及其种间杂交渐渗. 植 物生态学报 32, 704-718.

刘亚令, 李作洲, 张鹏飞, 姜正旺, 黄宏文 (2006). 猕猴桃自 然居群SSR遗传变异的空间自相关分析. 生物多样性 $\mathbf{1 4}$, 421-426.

王春国, 古瑜, 陈成涁, 焦定量, 薛振毅, 宋文芹 (2009). 不 同倍性西瓜基因组DNA甲基化水平与模式的MSAP分析. 分子细胞生物学报 42, 118-126.

薛梅, 陈成樹, 陈力, 马小军 (2008). 半夏多倍体复合体基因 
组DNA甲基化状态的MSAP分析. 中草药 39, 1713-1716. 张田, 李作洲, 刘亚令, 姜正旺, 黄宏文 (2007). 猕猴桃属植 物的cpSSR遗传多样性及其同域分布物种的杂交渐渗与同 塑. 生物多样性 15, 1-12.

Aversano R, Di Dato F, Di Matteo A, Frusciante L, Carputo $D$ (2011). AFLP analysis to assess genomic stability in Solanum regenerants derived from wild and cultivated species. Plant Biotechnol Rep 5, 265-271.

Beest M, Le Roux JJ, Richardson DM, Brysting AK, Suda J, Kubešová M, Pyšek P (2012). The more the better? The role of polyploidy in facilitating plant invasions. Ann Bot 109, 19-45.

Doyle JJ, Doyle JL (1987). A rapid DNA isolation procedure for small quantities of fresh leaf tissue. Phytochem Bull 19, 11-15.

Excoffier L, Laval G, Schneider S (2005). Arlequin ver. 3.0: an integrated software package for population genetics data analysis. Evol Bioinform Online 1, 47-50.

Falk DA, Holsinger KE (1991). Genetics and Conservation of Rare Plants. New York: Oxford University Press. pp. 6-8.

Ferguson AR, Huang HW (2007). Genetic resources of kiwifruit: domestication and breeding. Hortic Rev 33, 1121.

Gao LX, Geng YP, Li B, Chen JK, Yang J (2010). Genome-wide DNA methylation alterations of Alternanthera philoxeroides in natural and manipulated habitats: implications for epigenetic regulation of rapid responses to environmental fluctuation and phenotypic variation. Plant Cell Environ 33, 1820-1827.

Hardy OJ, Vekemans X (2001). Patterns of allozyme variation in diploid and tetraploid Centaurea jacea at different spatial scales. Evolution 55, 943-954.

Herrera CM, Bazaga P (2011). Untangling individual variation in natural populations: ecological, genetic and epigenetic correlates of long-term inequality in herbivory. Mol Ecol 20, 1675-1688.

Huang HW, Wang Y, Zhang ZH, Jiang ZW, Wang SM (2004). Actinidia germplasm resource and kiwifruit industry in China. Hortic Sci 39, 1165-1172.

Hulce D, Li X, Snyder-Leiby T, Johathan Liu CS (2011). GeneMarker® genotyping software: tools to increase the statistical power of DNA fragment analysis. J Biomol Tech 22, S35-S36.

Keyte AL, Percifield R, Liu B, Wendel JF (2006). Infraspecific DNA methylation polymorphism in cotton (Gossypium hirsutum L.). J Hered 97, 444-450.
Kolář $P$, Štech $M$, Trávníček $P$, Rauchová J, Urfus T, Vít P, Kubešová M, Suda J (2009). Towards resolving the Knautia arvensis agg. (Dipsacaceae) puzzle: primary and secondary contact zones and ploidy segregation at landscape and microgeographic scales. Ann Bot 103, 963974.

Li A, Hu BQ, Xue ZY, Chen L, Wang WX, Song WQ, Chen CB, Wang CG (2011). DNA methylation in genomes of several annual herbaceous and woody perennial plants of varying ploidy as detected by MSAP. Plant Mol Biol Rep 29, 784-793.

Li DW, Liu YF, Zhong CH, Huang HW (2010). Morphological and cytotype variation of wild kiwifruit (Actinidia chinensis complex) along an altitudinal and longitudinal gradient in central-west China. Bot J Linn Soc 164, 72-83.

Li XL, Yu XM, Wang NN, Feng QZ, Dong ZY, Liu LX, Shen JL, Liu B (2007). Genetic and epigenetic instabilities induced by tissue culture in wild barley (Hordeum brevisubulatum (Trin.) link). Plant Cell Tissue Organ Cult 90, 153-168.

Lira-Medeiros CF, Parisod C, Fernandes RA, Mata CS, Cardoso MA, Ferreira PCG (2010). Epigenetic variation in Mangrove plants occurring in contrasting natural environment. PLoS One 5, e10326.

Liu YF, Liu YL, Huang HW (2010). Genetic variation and natural hybridization among sympatric Actinidia species and the implications for introgression breeding of kiwifruit. Tree Genet Genome 6, 801-813.

Marhold K, Kudoh H, Pak JH, Watanabe K, Španiel S, Lihová J (2010). Cytotype diversity and genome size variation in eastern Asian polyploid Cardamine (Brassicaceae) species. Ann Bot 105, 249-264.

Masterson J (1994). Stomatal size in fossil plants: evidence for polyploidy in majority of angiosperms. Science 264, 421-424.

Mayfield D, Chen ZJ, Pires JC (2011). Epigenetic regulation of flowering time in polyploids. Curr Opin Plant Biol 14, 174-178.

Meirmans PG, Vlot EC, DenNijs JCM, Menken SBJ (2003). Spatial ecological and genetic structure of a mixed population of sexual diploid and apomictic triploid dandelions. J Evol Biol 16, 343-352.

Paun O, Bateman RM, Fay MF, Hedrén M, Civeyrel L, Chase MW (2010). Stable epigenetic effects impact adaptation in allopolyploid orchids (Dactylorhiza: Orchidaceae). Mol Biol Evol 27, 2465-2473.

Reyna-López GE, Simpson J, Herrera JR (1997). Differ- 
ences in DNA methylation patterns are detectable during the dimorphic transition of fungi by amplification of restriction polymorphisms. Mol Gen Genet 253, 703-710.

Rohlf FJ (2002). NTSYSpc: Numerical Taxonomy System Ver. 2.1. Setauket, NY: Exeter Publishing, Ltd.

Salmon A, Ainouche ML, Wendel JF (2005). Genetic and epigenetic consequences of recent hybridization and polyploidy in Spartina (Poaceae). Mol Ecol 14, 11631175.

Soltis DE, Buggs RJA, Doyle JJ, Soltis PS (2010). What we still don't know about polyploidy. Taxon 59, 13871403.

Suda J, Weiss-Schneeweiss H, Tribsch A, Schneeweiss GM, Trávníček P, Schönswetter P (2007). Complex distribution patterns of di-, tetra-, and hexaploid cytotypes in the European high mountain plant Senecio carniolicus (Asteraceae). Am J Bot 94, 1391-1401.

Vos P, Hogers R, Bleeker M, Reijans M, Van de Lee T, Hornes M, Frijters A, Pot J, Peleman J, Kuiper M, Zabeau M (1995). AFLP: a new technique for DNA-fingerprinting. Nucleic Acids Res 23, 4407-4414.

Xiong LZ, Xu CG, Maroof MA, Zhang QF (1999). Patterns of cytosine methylation in an elite rice hybrid and its parental lines, detected by a methylation-sensitive amplification polymorphism technique. Mol Gen Genet 261, 439446.

Yeh FC, Yang R, Boyle TJ, Xiyan JM (2000). PopGene32: Microsoft Window-based Freeware for Population Genetic Analysis, Ver.1.32. Edmonton, Alberta, Molecular Biology and Biotechnology Centre: University of Alberta.

\title{
Genetic and Epigenetic Variation in the Cytotype Mixture Population of Actinidia chinensis
}

\author{
Ling Yan $^{1,2 \uparrow}$, Yifei Liu ${ }^{3 \dagger}$, Hongwen Huang ${ }^{3 *}$ \\ ${ }^{1}$ Wuhan Botanical Garden, Chinese Academy of Sciences, Wuhan 430074, China; ${ }^{2}$ Graduate University of Chinese Aca- \\ demy of Sciences, Beijing 100049, China; ${ }^{3}$ Key Laboratory of Plant Resources Conservation and Sustainable Utilization, \\ South China Botanical Garden, Chinese Academy of Sciences, Guangzhou 510650, China
}

\begin{abstract}
The formation and maintenance of the cytotype mixture in plant natural populations has been found related to genetic and epigenetic variations of genomes. We investigated the patterns of genetic variation, genetic structure and methylation levels, as well as epigenetic differentiation, of a cytotype mixture population of the Actinidia chinensis complex by amplified fragment-length polymorphism (AFLP) and methylation-sensitive amplification polymorphism (MSAP). The mixture population had high genetic and epigenetic diversity. The relationship between population genetic diversity and altitude but not epigenetic diversity and altitude was significant $(P<0.05)$. As well, patterns of genetic and epigenetic variation were not positively correlated. Analysis of molecular variance revealed that most genetic and epigenetic variations occurred within ploidy races $(97.65 \%$ vs $99.84 \%, P<0.05)$. Neighbor-joining tree analysis based on AFLP analysis revealed that the same ploidy individuals clustered preferentially, with no obvious cluster based on ploidy levels in the MSAP tree. The total methylation level in the cytotype mixture population was $24.86 \%$. Moreover, tetraploids and hexaploids showed more variation than diploids in genomic methylation, including increase and loss of methylation loci. We provide a theoretical basis to understand the formation and maintenance mechanism in the cytotype mixture population of A. chinensis.
\end{abstract}

Key words Actinidia chinensis, cytotype mixture, DNA methylation, genetic structure, polyploidy

Yan L, Liu YF, Huang HW (2012). Genetic and epigenetic variation in the cytotype mixture population of Actinidia chinensis. Chin Bull Bot 47, 454-461.

\footnotetext{
$\dagger$ These authors contributed equally to this paper.

*Author for correspondence. E-mail: huanghw@mail.scbg.ac.cn
} 